\title{
MicroRNA-20b promotes cell proliferation via targeting of TGF- $\beta$ receptor II and upregulates MYC expression in Ewing's sarcoma cells
}

\author{
MASANORI KAWANO, KAZUHIRO TANAKA, ICHIRO ITONAGA, TATSUYA IWASAKI and HIROSHI TSUMURA \\ Department of Orthopaedic Surgery, Faculty of Medicine, Oita University, Oita 879-5593, Japan
}

Received February 10, 2017; Accepted September 6, 2017

DOI: $10.3892 /$ ijo.2017.4155

\begin{abstract}
Transforming growth factor- $\beta$ receptor II (TGFBR2) is implicated in various types of cancer. Most molecules involved in the TGF- $\beta$ pathway can be degraded by one or more microRNAs (miRNAs). In the present study, we show that miRNA plays an important role in downregulating TGFBR2 expression in Ewing's sarcoma (ES) cells. Microarray-based analyses revealed that the expression of miR-20b was significantly increased, whereas TGFBR2 and MYC were significantly downregulated and upregulated, respectively, in all ES cells compared to their expression in human mesenchymal stem cells (hMSCs). In ES cell lines, anti-miR-20b increased TGFBR2 expression and significantly decreased MYC expression, showing an inverse relationship with TGFBR2. The induction by anti-miR-20b further prohibited ES cell growth and cell cycle progression. Moreover, decreased miR-20b in ES cells significantly inhibited tumor growth in vivo. Taken together, these results suggest that miR-20b behaves as an oncogene in ES when its overexpression is unregulated by targeting TGFBR2. Because downstream TGFBR 2 and TGF- $\beta$ signaling regulate cell cycle, apoptosis, and tumor proliferation via MYC, our findings may contribute to new targeted therapies for ES.
\end{abstract}

\section{Introduction}

Ewing's sarcoma (ES) bearing a pathognomonic Ewing sarcoma protein-friend leukemia integration factor 1 translocation is the second most common bone tumor in children and adolescents (1). The current standard of care combines a 5-drug chemotherapy regimen (vincristine, doxorubicin, cyclophosphamide, ifosfamide and etoposide) and local control comprising tumor resection and/or radiation therapy (2). However, the high percentage of relapse and metastasis and

Correspondence to: Dr Kazuhiro Tanaka, Department of Orthopaedic Surgery, Faculty of Medicine, Oita University, Oita 879-5593, Japan

E-mail:ktanaka@oita-u.ac.jp

Key words: Ewing's sarcoma, microRNA, miR-20b, transforming growth factor- $\beta$ receptor II, MYC the poor prognosis of patients with ES have led to renewed interest in novel treatment.

miRNAs are known to be involved in cancer progression. Functionally, aberrant miRNA expression can affect cell biology $(3,4)$. Recent studies have shown that miR-20b is significantly upregulated in various types of cancer (5-7); however, the role of miR-20b in the proliferation of ES cells remains unknown.

Transforming growth factor- $\beta$ receptor II (TGFBR2), as a tumor suppressor (8), is downregulated in various types of cancer and is generally involved in cancer development $(9,10)$. Most cancer cells lack sensitivity to TGF- $\beta$-mediated growth inhibitory responses upon TGFBR2 downregulation (11). Recently, several reports have demonstrated that TGFBR2 is involved in cell proliferation, migration and invasion in gastric cancer via several transcriptional factors including MYC (12).

In the present study, we explored the genome-wide expression of both miRNAs and mRNAs in human mesenchymal stem cells (hMSCs) and five human ES cell lines. The results showed that miR-20b expression was increased, while that of TGFBR2 was repressed and MYC was upregulated in five ES cell lines compared to in hMSCs. We hypothesized that the influence of TGFBR2 in ES cells is mediated, at least in part, either directly or indirectly by miR-20b and that MYC expression increases in response to the downstream effector of Smad. The aim of this study was to evaluate whether MYC expression is induced by miR-20b via TGFBR 2 inhibition and if this pathway plays a role in the malignancy of ES cells.

\section{Materials and methods}

Cell lines and reagents. Human MSC (hMSCs) was obtained from Takara Bio, Inc. (Shiga, Japan). Human ES cells (SK-N-MC, RD-ES, SK-ES-1 and SCCH) were purchased from the Japanese Collection of Research Bioresources (Tokyo, Japan). WE-68, a human ES cell line, was generously provided by Professor Frans van Valen (Westfalische-Wilhelms University, Munster, Germany). High glucose-Dulbecco's modified Eagle's medium (DMEM), RPMI-1640 medium, minimal essential medium (MEM), and fetal bovine serum (FBS) were purchased from Invitrogen (Carlsbad, CA, USA). Mesenchymal Stem Cell (MSC) Basal Medium, Chemically defined (MSCBM-CD) and MSCGM-CD SingleQuats were obtained from Takara Bio. 
The RNeasy kit and miRNeasy Mini kit were obtained from Qiagen (Hilden, Germany) and TRIzol reagent was from Invitrogen. The miR-20b-5p mimic (5'-CAAAGUGCUCAU AGUGCAGGUAG-3'), miR-20b-5p mutant (5'-CUUUCAC GUCAUAGUGCAGGUAG-3'), hsa-miR-20b inhibitor and negative control (NC) miRNAs were purchased from Invitrogen. The transfection reagent Lipofectamine 2000 and antibiotics-free Opti-MEM were also obtained from Invitrogen. Actinomycin D was from Sigma-Aldrich (St. Louis, MO, USA). The TGFBR2 expression plasmid (SC119965) was obtained from Origene Technologies Inc. (Rockville, MD, USA).

Antibodies produced in rabbits for TGFBR2 (\#9552), MYC (\#9402), $\beta$-actin (\#4970), Akt (\#4691), phosphorylated-Akt (p-Akt) (\#4060), p21 (\#2947), Smad2/3 (\#8685), phosphorylated-Smad2/3 (p-Smad2/3) (\#8828), PAR/poly(ADP-ribose) polymerase (PARP) (\#9542), and cleaved PARP (\#9541) were purchased from Cell Signaling Technology (Danvers, MA, USA) and phosphorylated-p21 (p-p21) (ab47300) was from Abcam (Cambridge, UK). Horseradish peroxidase-conjugated anti-rabbit immunoglobulin $\mathrm{G}$ antibodies and the ECL Prime system were obtained from GE Healthcare (Little Chalfont, UK). The Cycletest Plus DNA reagent kit and Annexin V-FITC apoptosis detection kit were obtained from BD Biosciences (Franklin Lakes, NJ, USA).

BALB/c nu/nu nude mice were purchased from Kudo (Tosu, Japan). All protocols for animal experiments in the present study were approved by the Ethics Review Committee for Animal Experimentation of Oita University.

Cell culture. SK-N-MC and RD-ES cells were cultured in DMEM high-glucose medium and SK-ES1 and WE-68 cells were cultured in RPMI-1640 medium. The media were supplemented with $10 \%$ FBS and $1 \%$ penicillin/streptomycin. SCCH cells were cultivated in MEM supplemented with $10 \%$ FBS and $0.1 \mathrm{mM}$ non-essential amino acids. hMSCs were maintained in MSCBM-CD supplemented with SingleQuats.

The cells were incubated at $37^{\circ} \mathrm{C}$ in an incubator chamber supplemented with $5 \% \mathrm{CO}_{2}$ and passaged when the cells were $\sim 70 \%$ confluent.

miRNA and mRNA expression analysis using microarray. miRNA and total RNA were extracted using miRNeasy and RNeasy kits, respectively, from the cells according to the manufacturer's recommendations. The quality of RNA was confirmed using a Bioanalyzer 2100 (Agilent Technologies, Santa Clara, CA, USA).

An aliquot $(1 \mu \mathrm{g})$ of the RNA fraction including miRNAs from each of the five ES cells and hMSCs was biotin-labeled with the FlashTag Biotin HSR kit (Genisphere LLC, Hatfield, PA, USA) and subjected to miRNA expression array analyses with a GeneChip miRNA 3.0 array (Affymetrix, Santa Clara, CA, USA). The array data were quantile normalized, log2transformed using miRNA QC software (Affymetrix) and analyzed using GeneSpring GX 11.0 (Agilent Technologies).

For mRNA expression analysis, 1 ng total RNA from each of five ES cells and hMSCs was used to generate doublestranded cDNA by reverse transcription, and then used to generate biotinylated cRNA by in vitro transcription using the 3' IVT Express kit (Affymetrix). The cRNA probes were hybridized to the GeneChip Genome HG U133 Plus 2.0 array (Affymetrix). GeneSpring GX 11.0 software was used for array analyses including normalization and filtering (20.0-100.0th percentile). All experiments were repeated twice. Variant analyses were carried out to determine the significance of differences between two groups. Genes showing a 2-fold or more significant increase or reduction in expression were determined and evaluated using the KEGG pathway database (http://www.genome.jp/kegg/pathway.html) for pathway analyses.

Target prediction of miRNAs. To predict miRNA target genes, microRNA.org (http://www.microrna.org/), TargetScan 6.0 (http://www.targetscan.org/), Basic Local Alignment Search Tool (BLAST), DIANA tools (http://diana.imis.athena-innovation.gr/DianaTools/) and PicTar (http://pictar.mdc-berlin. $\mathrm{de} /$ ) were used. The results of database analyses suggested that TGFBR2 was the strongest target of miR-20b.

Transfection and cell proliferation analysis. Cells $\left(1 \times 10^{5}\right)$ were cultured in $2 \mathrm{ml}$ medium without antibiotics in 6-well plates. miR-20b-5p mimic, miR-20b-5p mutant, anti-miR-20b inhibitor, negative control (NC) miRNAs, TGFBR2 expression vector, and Mock vector were transfected using Lipofectamine 2000. Additionally, $10 \mu \mathrm{g} / \mathrm{ml}$ of actinomycin D was used to inhibit de novo RNA transcription. The transfected cells were incubated for $48 \mathrm{~h}$ and subjected to further analyses.

The number of viable cells was counted with a TC10 Automated Cell Counter (Bio-Rad Laboratories, Hercules, CA, USA). The cell cycle distribution was monitored by propidium iodide (PI) staining using a fluorescence activated cell sorting (FACS) analyzer FACSVerse (BD Biosciences). The number of cells in the cell cycle phases of G0/G1, S and G2/M was determined. All experiments were performed in triplicate.

Quantitative RT-PCR. The transfected cells were harvested and lysed using TRIzol reagent to extract total RNA. cDNA was generated and quantitative real-time PCR (qRT-PCR) was carried out using a LightCycler 480 (Roche, Basel, Switzerland). The relative expression of TGFBR2 and GAPDH was calculated using the $2^{-\Delta \Delta \mathrm{Ct}}$ method. The following primers were used: TGFBR2-forward, 5'-AAGACAAAGCCAACCGAT AC-3' and TGFBR2-reverse, 5'-GAAGTTGAACTGCTAGC CTC-3'; GAPDH-forward, 5'-CCTCTATGCCAACACAGT GC-3' and GAPDH-reverse, 5'-GTACTCCTGCTTGCTGAT CC-3'.

Western blotting. Cellular proteins were extracted and an aliquot $(15 \mu \mathrm{g})$ was applied to a $10 \%$ Tris- $\mathrm{HCl}$ Criterion precast gel (Bio-Rad Laboratories). Proteins in the gel were transferred onto polyvinylidene fluoride membranes and reacted with the anti-TGFBR2, Smad2/3, p-Smad2/3, Akt, p-Akt, p21, p-p21, MYC and $\beta$-actin antibodies. The blots were treated with anti-rabbit IgG antibodies and signals were detected using the ECL Prime system (GE Healthcare). Protein expression was quantified using ImageQuant TL software (GE Healthcare). All experiments were conducted in triplicate.

Detection of apoptosis. Apoptotic cells were detected by FACS analysis and western blotting. SK-ES-1 cells $\left(1 \times 10^{6}\right.$ 
A

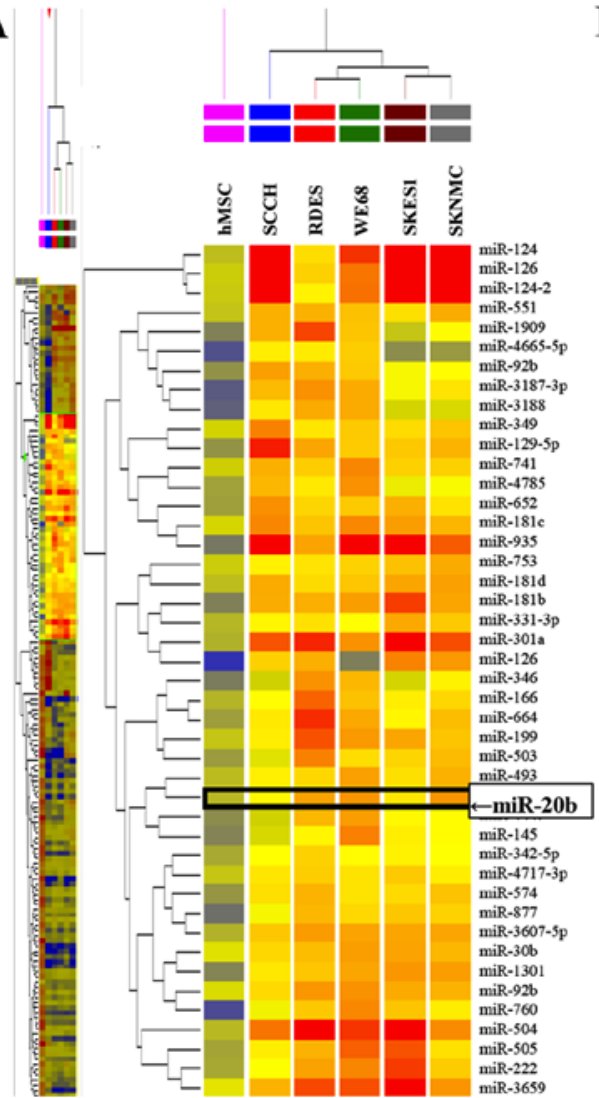

$\mathbf{B}$

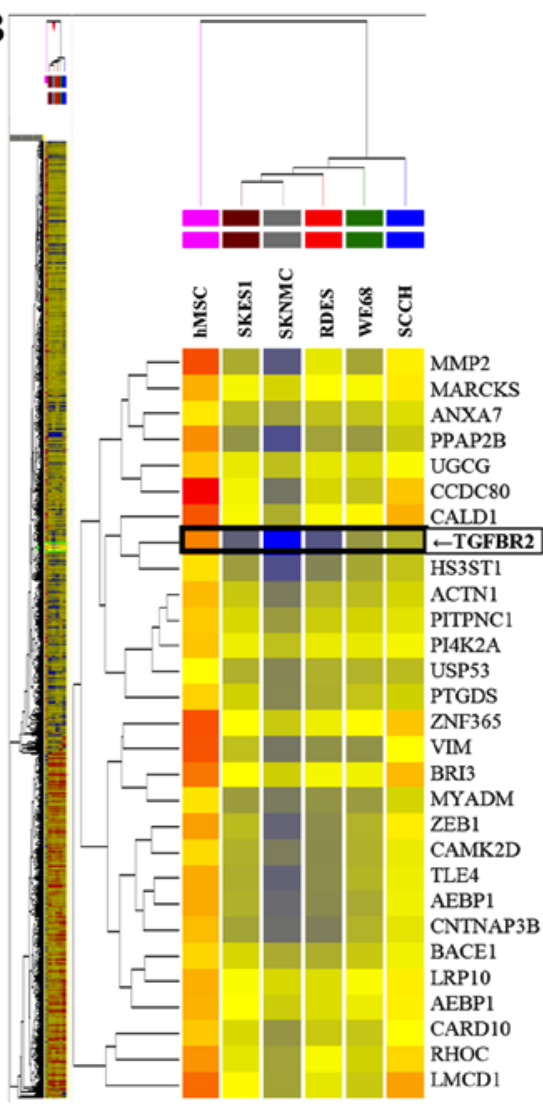

C

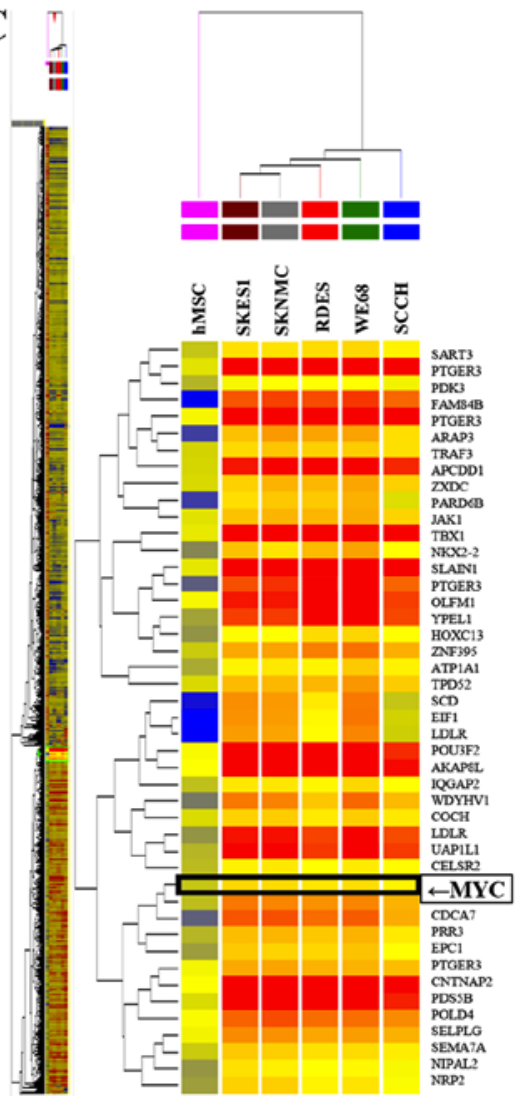

Figure 1. Whole genome array analysis in ES cell lines. (A) miRNA expression in five ES cell lines (SCCH, RDES, WE68, SK-ES-1 and SKNMC) and hMSCs. Heat maps of mRNA expression and extraction of TGFBR2 (B) and MYC (C) in ES cells and hMSCs. The color bar shows the relative expression levels; red and blue indicate an increase and decrease, respectively.

cells) were cultured and transfected with the anti-miR-20b miRNA or TGFBR2 expression vector. Forty-eight hours after the transfection, the cells were analyzed using FACSVerse to detect cell death. As a positive control for apoptosis, the cells were treated with a low dose $(5 \mu \mathrm{g} / \mathrm{ml})$ of doxorubicin. The expression of apoptosis-related proteins, including PARP and cleaved PARP, were analyzed by western blotting.

In vivo experiments using nude mice. SK-ES-1 cells $\left(2 \times 10^{6}\right)$ transfected with anti-miR-20b were suspended in $100 \mu 1$ normal saline and injected into the gluteal region of $\mathrm{BALB} / \mathrm{c}$ $\mathrm{nu} / \mathrm{nu}$ mice. A total of 28 mice were divided into four groups (7 mice each): i) untreated control; ii) transfected with NC-miRNA; iii) transfected with anti-miR-20b; and iv) transfected with TGFBR2 expression vector. Changes in the weight of treated mice and tumor volume were monitored for 6 weeks. Tumor volume was calculated using the formula: $\mathrm{V}=\left(\right.$ length $\mathrm{x}$ width $\left.{ }^{2}\right) / 2$. The mice were sacrificed, and xenografted tumors were removed and subjected to immunohistochemistry. Resected tumors were fixed with $4 \%$ formaldehyde, paraffin-embedded, sectioned using a microtome, and reacted with anti-TGFBR2 and MYC antibodies. The expression of proteins in the section was visualized using the DAB and EnVision System (Dako, Glostrup, Denmark).

Statistical analysis. Two-tailed Student's t-test was carried out for continuous variables. Differences among $>3$ groups were analyzed using analysis of variance and Scheffe test. The results were expressed as the mean \pm standard deviation (SD), and differences were considered significant when P-values were $<0.05$. All statistical analyses were conducted using the SPSS 18.0 software (SPSS, Inc., Chicago, IL, USA).

\section{Results}

Expression of miR-20b in ES cells. To determine the expression profiles of miRNAs in ES cell lines, array analysis was carried out. The results demonstrated that 1054 miRNAs in ES cells showed significantly altered expression ( $>2$-fold change) compared to in hMSCs (Fig. 1A). The expression of 228 miRNAs was significantly increased, while that of 705 was significantly decreased in all ES cell lines tested. The remaining 121 miRNAs exhibited different expression patterns among the five types of ES cells. Among the 228 upregulated miRNAs in five ES cells, the expression of miR-20b was increased by 2.36- to 5.7 -fold compared to in hMSCs.

Decrease in TGFBR2 and MYC expression in ES cells. The expression profiles of mRNAs in ES cell lines were then analyzed using a cDNA array. The data demonstrated that 3043 mRNAs in ES cells exhibited significantly different expression from those in hMSCs. The expression of 1062 mRNAs was significantly increased, while that of 1884 was significantly decreased in all ES cell lines tested. The remaining 97 mRNAs showed different expression patterns among the five types of ES cells. Among the 1884 downregulated mRNAs in five ES 
A Chromosome 3

(IIIIIIIIII)

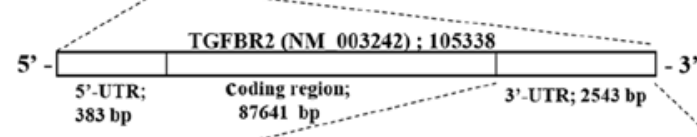

3' gauggacgugauacuCgugaAac 5' hsa-miR-20b

$:|| \quad:|||\quad|||||||$

253:5' uagcCAAUAACAUUUGCACUUUa 3 ' TGFBR2

hsa-miR-20b-5p sequence;

WT; 3'- GAUGGACGUGAUACUCGUGAAAC-5'

hsa-mir-20b-5p mutant sequence;

Mut; 3'- GAUGGACGUGAUACUGCACUUUC-5'
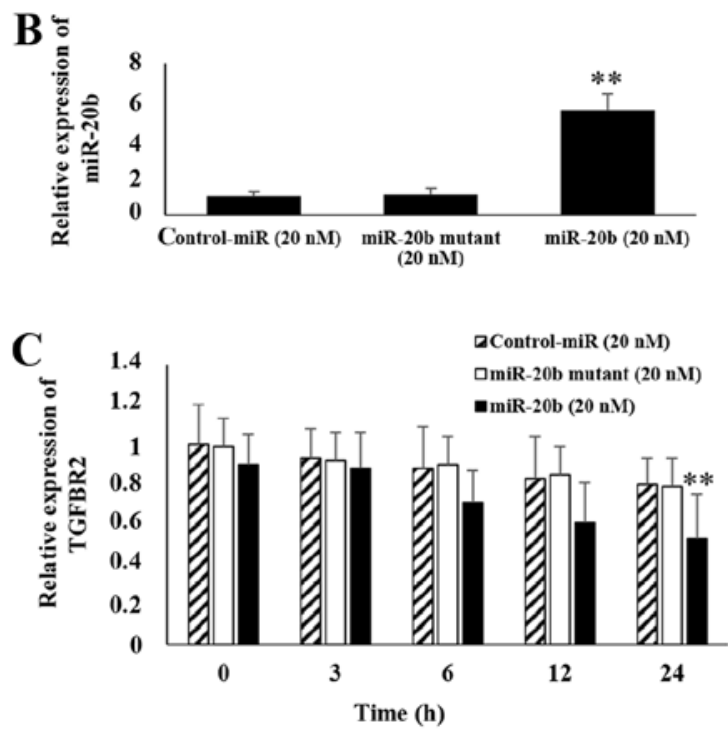

D
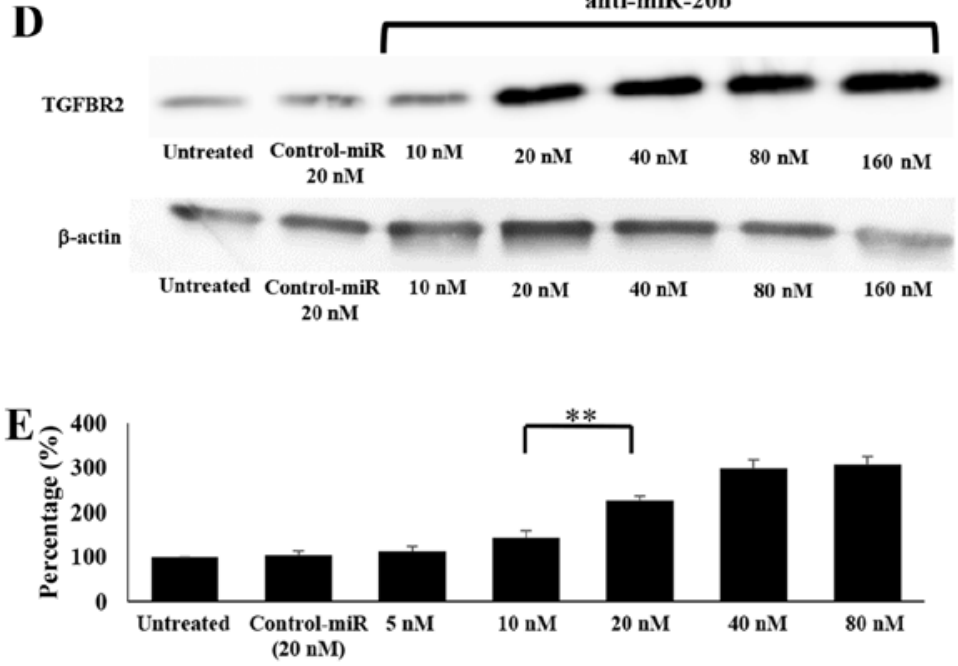

$\mathbf{F}$
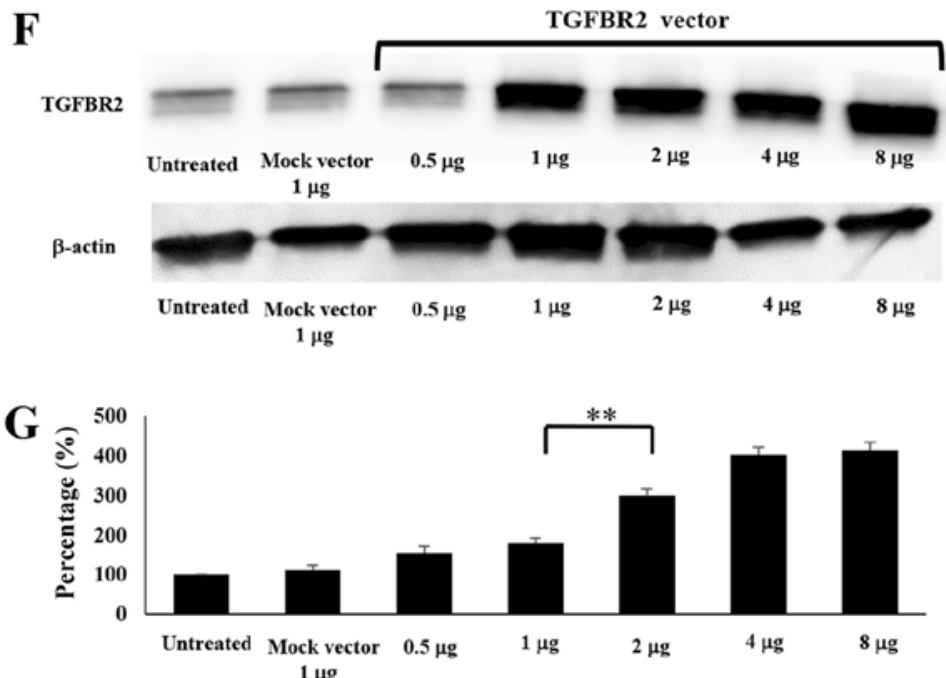

$1 \mu \mathrm{g}$

Figure 2. Inhibition by miR-20b of TGFBR 2 mRNA expression and repressing of TGFBR2 with anti-miR-20b in SKES1 cells. (A) Possible binding sites of miR-20b at the 3'-UTR site of TGFBR2 mRNA. Each sequence of miR-20b (Wt) and its mutant (Mut). (B) After actinomycin D administration, the miR-20b (B) and mRNA (C) expression levels (B) in the negative control-miR, miR-20b and miR-20b mutant were evaluated by qRT-PCR. (D) Western blot analysis showed increased TGFBR2 protein in anti-miR-20b and TGFBR2-expression vector group. (E) Densitometry evaluation of TGFBR2 protein. (F) Western blot analysis showing increased expression of TGFBR2 protein following transfection of TGFBR2 expression vector in SKES1 cells. (G) Expression of TGFBR2 protein after induction of TGFBR2 vector. ${ }^{* *} \mathrm{P}<0.01$.

cells, the expression of TGFBR2 (Fig. 1B) was decreased by 2.38- to 3.29-fold compared to in hMSCs.

TGFBR 2 as a direct target of miR-20b in ES cells. BLAST and TargetScan analyses revealed considerable complementarity in the sequence of the miR-20b seed region with the human TGFBR2 mRNA 3' untranslated region (3'-UTR) (Fig. 2A), suggesting that miR-20b influences TGFBR 2 mRNA levels by associating with the 3 '-UTR of the mRNA. Therefore, we examined the effects of miR-20b on the expression of TGFBR 2 in ES cells by transfecting miR-20b and a mutated miR-20b into SK-ES-1 cells. In this experiment, de novo mRNA transcription was blocked by actinomycin D $(10 \mu \mathrm{g} / \mathrm{ml})$, an inhibitor of mRNA transcription, as we attempted to determine whether TGFBR 2 mRNA stability was affected by miR-20b. Using a microRNA mutant oligonucleotide method rather than the luciferase method, we found that the microRNA disrupted and/or interfered with target mRNA expression (13-15). We observed an increase in the intracellular miR-20b level by 5.51-fold compared to control-miR (Fig. 2B) and significantly decreased TGFBR 2 expression by 0.53 -fold at the mRNA level after transfection with the miR-20b oligonucleotide (Fig. 2C). The results suggest that the stability of TGFBR 2 mRNA was inhibited by miR-20b in ES cell lines.

Effects of anti-miR-20b on expression of TGFBR2. We next examined the effects of anti-miR-20b oligonucleotide, an inhibitor of miR-20b, on the expression of TGFBR2 in SK-ES-1 cells. In anti-miR-20b-transfected cells, the levels of TGFBR2 protein were remarkably elevated compared to in untreated or control oligonucleotide-treated cells (Fig. 2D). The level of TGFBR2 protein expression in cells transfected with antimiR-20b (20 nM) was upregulated by 2.26 -fold compared to in control cells $(\mathrm{P}<0.01$; Fig. $2 \mathrm{E})$. Western blot analyses further demonstrated that the levels of TGFBR2 protein in TGFBR2 expression vector-transfected cells were significantly increased 
A $\left(\mathbf{x 1 0 ^ { 4 } \text { cells } )}\right.$
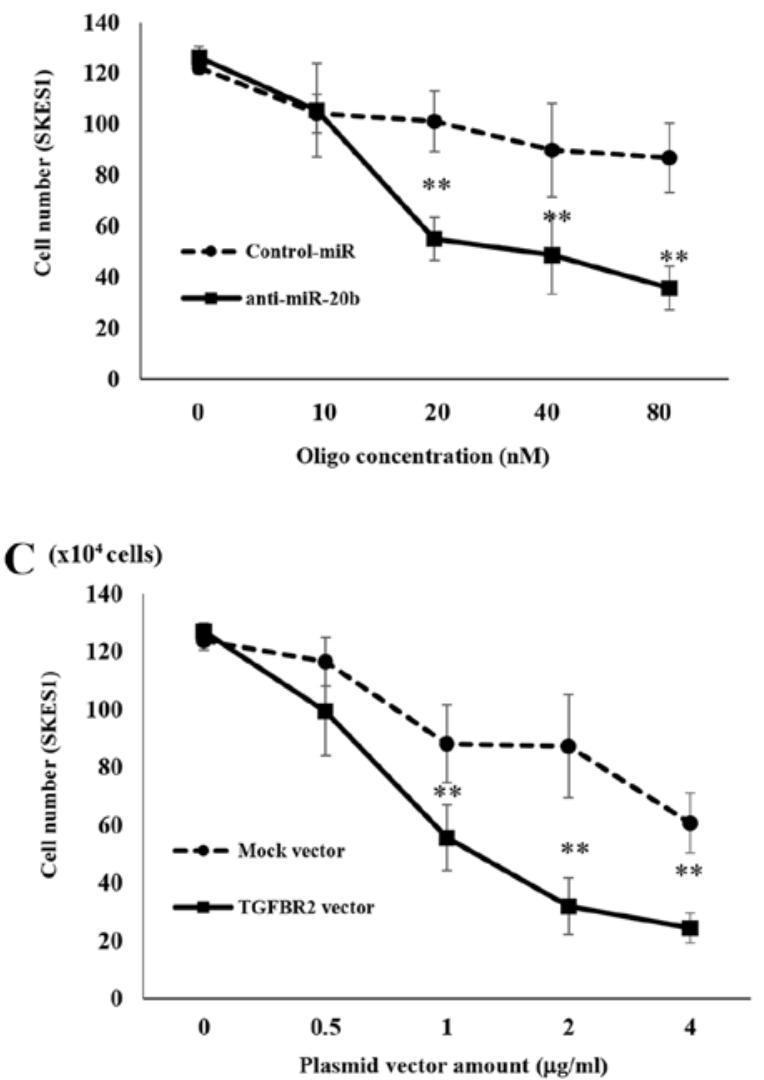
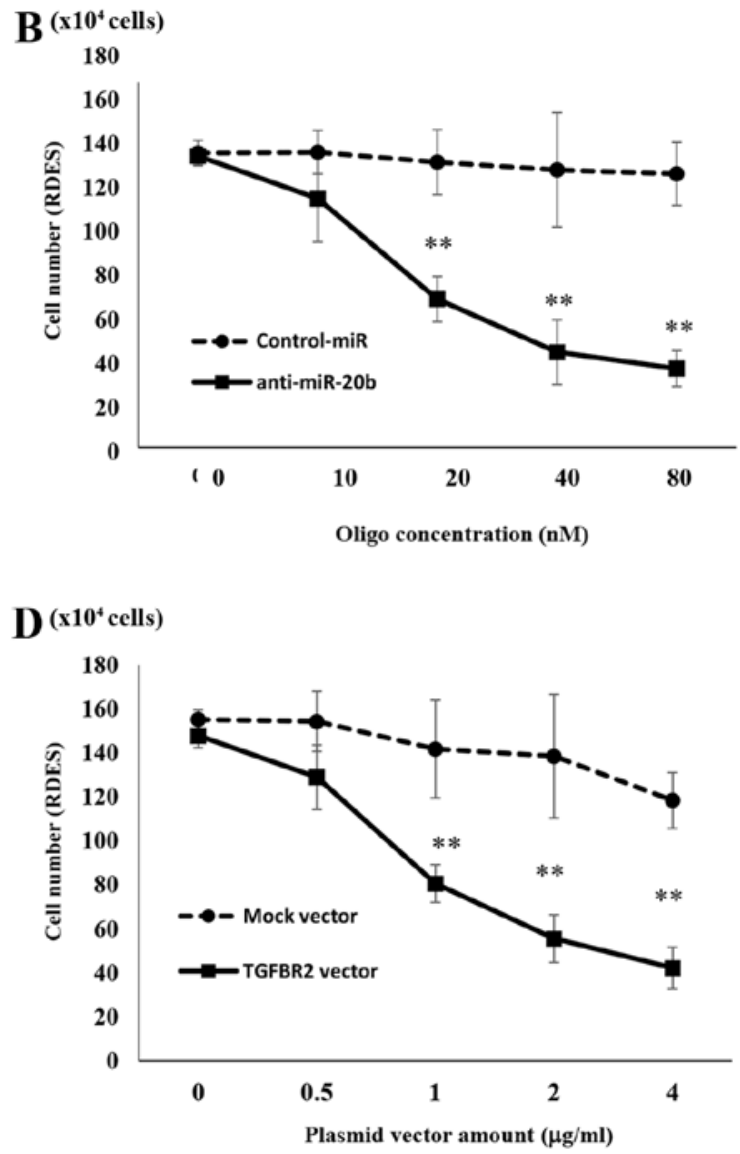

Figure 3. Cell growth assay of the effects of anti-miR-20b. Anti-miR-20b inhibits cell growth of SK-ES-1 cells (A) and RDES cells (B). TGFBR2 expression vector inhibits cell growth of SK-ES-1 cells (C) and RDES cells (D). Data represent the mean \pm SD of three independent experiments. $P<0.05$ was considered to indicate significance: ${ }^{*} \mathrm{P}<0.05,{ }^{* *} \mathrm{P}<0.01$.

compared to those in mock vector-transfected cells (Fig. 2F). Compared with the control, cells transfected TGFBR2 vector $(1 \mu \mathrm{g})$ showed significantly increased expression of TGFBR2 protein by 2.98 -fold $(\mathrm{P}<0.01$; Fig. $2 \mathrm{G})$.

Inhibition of ES cell proliferation by anti-20b-miR and $T G F B R 2$. It is well-known that TGFBR2 plays a pivotal role in controlling cell cycle progression. To examine the effects of TGFBR 2 on proliferation of ES cells, a TGFBR2 expression vector was transfected into SK-ES-1 cells. Because introduction of anti-miR-20b led to decreased expression of TGFBR2, we also investigated the effects of anti-miR-20b on ES cell growth. Compared to Control-miRNA-transfected cells $\left(10.1 \pm 1.18 \times 10^{5}\right.$ cells $)$, anti-miR-20b-transfected SK-ES-1 cells showed a significant decrease in the cell number $\left(5.5 \pm 0.84 \times 10^{5}\right.$ cells $)$ at $48 \mathrm{~h}$ after transfection (Fig. 3A). Treatment with anti-miR-20b also inhibited the proliferation of RD-ES cells $\left(7.3 \pm 1.12 \times 10^{5}\right.$ cells $)$ compared to in Control-miRNA-transfected cells $\left(11.4 \pm 1.6 \times 10^{5}\right.$ cells $)$ (Fig. 3B). TGFBR2 expression vector-transfected SK-ES-1 cells also showed decreased cell growth $\left(5.57 \pm 1.23 \times 10^{5}\right.$ cells $)$ compared to untreated cells or those transfected with the mock vector $\left(9.81 \pm 1.34 \times 10^{5}\right.$ cells) (Fig. 3C). Furthermore, compared to RD-ES cells transfected with mock vector $\left(12.12 \pm 2.22 \times 10^{5}\right.$ cells), cell growth was significantly decreased in RD-ES cells transfected with the TGFBR2 expression vector $\left(8.06 \pm 0.8 \times 10^{5}\right.$ cells) (Fig. 3D).
Association of TGFBR2 restoration and MYC expression. Since MYC was reported to be involved in the signaling pathway of TGFBR2 (12), we next examined the expression of TGFBR2, MYC and their downstream factors in SK-ES-1 cells (Fig. 4A) and RDES cells (Fig. 4B). When SK-ES-1 cells were transfected with the anti-miR-20b, the expression of TGFBR2 $(223 \pm 12.2 \%)$, phosphorylated-Smad2 $(189 \pm 3.9 \%)$, and phosphorylated-Smad3 $(287 \pm 15.6 \%)$ was dramatically increased compared to in control cells (100\%). When SK-ES-1 cells were transfected with the TGFBR 2 expression vector, the expression of TGFBR2 $(219 \pm 9.1 \%)$, phosphorylated-Smad2 $(199 \pm 3.1 \%)$ and phosphorylated-Smad3 $(299 \pm 16.5 \%)$ was dramatically increased compared to in control cells (100\%). TGFBR2 expression in RDES cells was increased transfected with the anti-miR-20b $(255 \pm 7.7 \%)$ and TGFBR2 vector $(250.1 \pm 8.8 \%)$ compared to in control cells (100\%). In contrast, MYC expression in SK-ES-1 cells was inhibited when transfected with the anti-miR-20b $(61.2 \pm 8.1 \%)$ and TGFBR2 vector $(54.2 \pm 1.9 \%)$ compared to in control cells $(100 \%)$. When RDES cells were transfected with the anti-miR-20b $(55 \pm 10.6 \%)$ or TGFBR2 expression vector $(33.2 \pm 6.8 \%)$, the expression of MYC was dramatically decreased compared to in control cells $(100 \%)$. Western blot analysis further demonstrated that the introduction of the anti-miR-20b into SK-ES-1 cells resulted in a reduction of $\mathrm{p}-\mathrm{Akt}(72 \pm 4.8 \%)$ and induction of p15 $(195 \pm 9.9 \%)$, p21 $(244 \pm 8.8 \%)$ and p27 $(263 \pm 5.5 \%)$ protein expression compared to untreated cells $(100 \%)$. The introduc- 

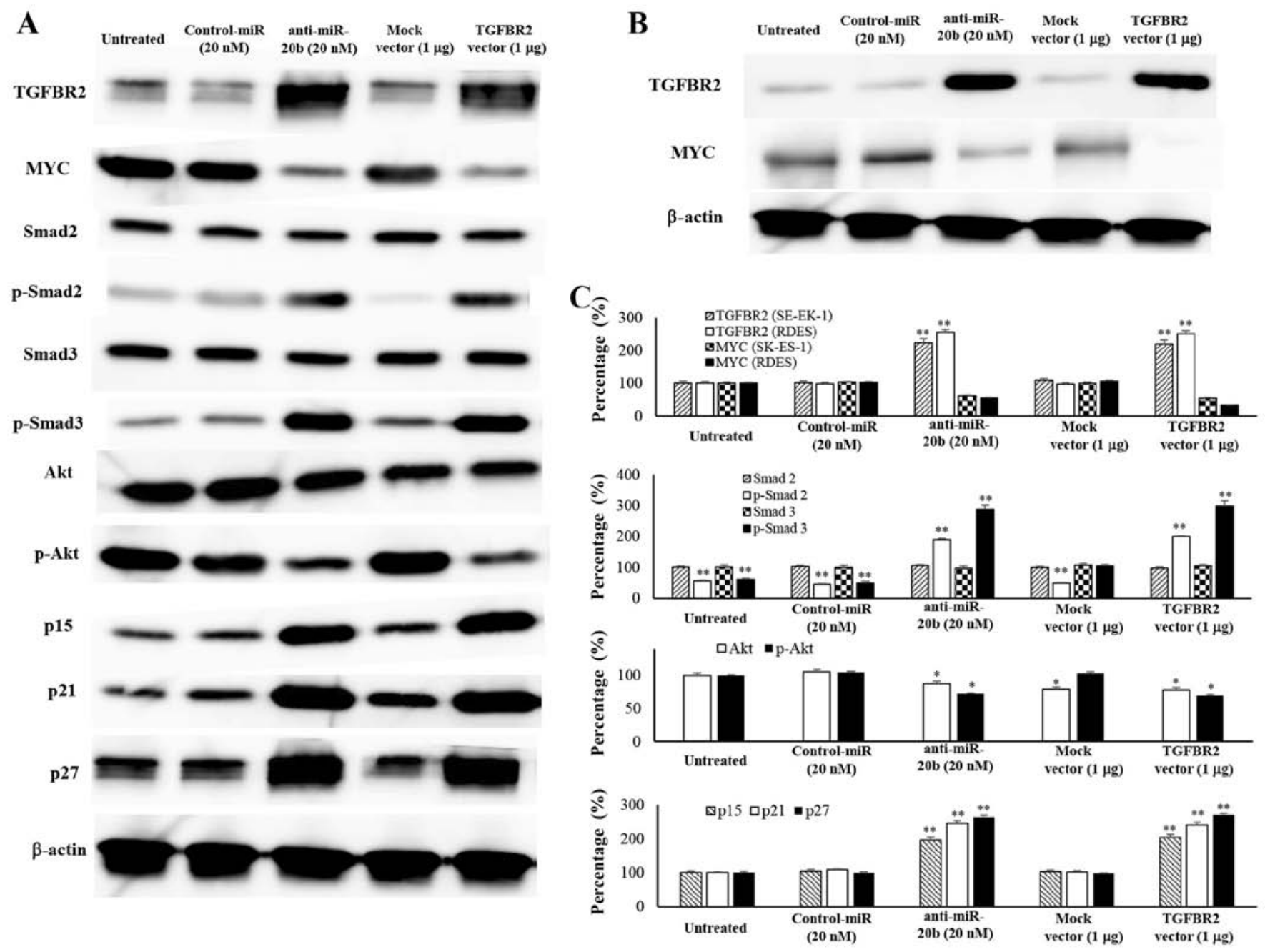

Figure 4. Immunoblot analysis of TGFBR2, MYC and TGF- $\beta$ signaling-related factors. Effects of anti-miR-20b and TGFBR2 vector on TGFBR2, MYC and TGF- $\beta$ pathway in (A) SK-ES-1 cells and (B) RDES. (C) The quantification of western blot analysis. Data represent the mean \pm SD of three independent experiments. $\mathrm{P}<0.05$ was considered to indicate significance: ${ }^{*} \mathrm{P}<0.05,{ }^{* *} \mathrm{P}<0.01$.

tion of the TGFBR2 expression vectors into SK-ES-1 cells resulted in a reduction of p-Akt $(77 \pm 1.5 \%)$ and induction of p15 $(204 \pm 9.2 \%)$, p21 $(239 \pm 8.4 \%)$ and p27 $(270 \pm 4.2 \%)$ protein expression compared to untreated cells (100\%) (Fig. 4C).

Cell cycle inhibition by anti-miR-20b and TGFBR2. To elucidate whether growth inhibition of ES cells is mediated by cell cycle retardation or apoptosis induction, we analyzed the cell cycle distribution of anti-miR-20b or TGFBR2 expression vector-treated cells (Fig. 5A-D). FACS analyses revealed that the number of SK-ES-1 cells transfected with anti-20b-miR or TGFBR2 vector in G0/G1 was significantly higher than that in control cells, whereas that in $\mathrm{G} 2 / \mathrm{M}$ phase was significantly lower than in controls (Fig. 5E). These results indicate that miR-20b and TGFBR2 have opposite effects on ES cell cycle progression. We next surveyed the expression of PARP and cleaved-PARP in SK-ES-1 cells. In anti-miR-20b- and TGFBR2-transfected cells, the expression of cleaved-PARP, an indicator of caspase-mediated apoptosis, was much lower than that in control cells. When a low dose of ADM was added to the cells, cleaved PARP expression was observed both in anti-20b-miR- and TGFBR2-transfected cells (Fig. 5F). When SK-ES-1 cells were treated with the anti-miR-20b and low dose AMD, the expression of cleaved PARP $(156 \pm 7.1 \%)$ was dramatically increased compared to in untreated cells (100\%). SK-ES-1 cells were treated with the TGFBR2 expression vector and low dose AMD, the expression of cleaved PARP $(166 \pm 6.6 \%)$ was dramatically increased compared to in untreated cells (100\%) (Fig. 5G). FACS analyses by double staining with Annexin V-FITC and PI also revealed no significant differences between the distribution patterns of anti-miR-20b or TGFBR2 vectorintroduced cells and control cells. In contrast, the induction of apoptosis was observed in SK-ES-1 cells transfected with the anti-miR-20b or TGFBR2 vector following treatment with low-dose ADM (Fig. 5H).

Growth inhibition of xenografted tumors by anti-20b-miR and TGFBR2. We next examined the effects of miR-20b inhibitor on the growth of xenografts in vivo. Introduction of anti-miR-20b into SKES1 cells resulted in the decreased growth of subcutaneous xenografted tumors in nude mice (Fig. 6A). The size of tumors in mice inoculated with antimiR-20b-transfected SK-ES-1 cells $\left(466.5 \pm 28.1\right.$ cells $\left./ \mathrm{mm}^{3}\right)$ 


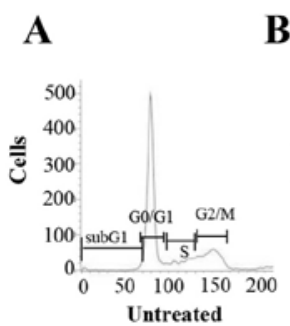

B

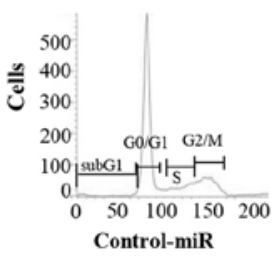

C

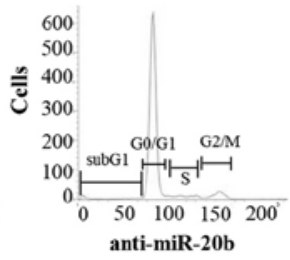

D

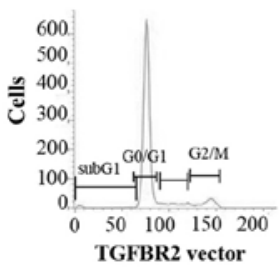

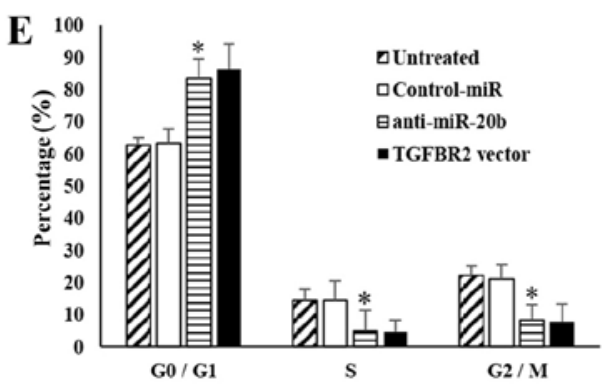

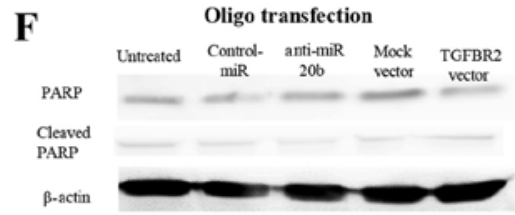

$\mathbf{H}$ Oligo transfection
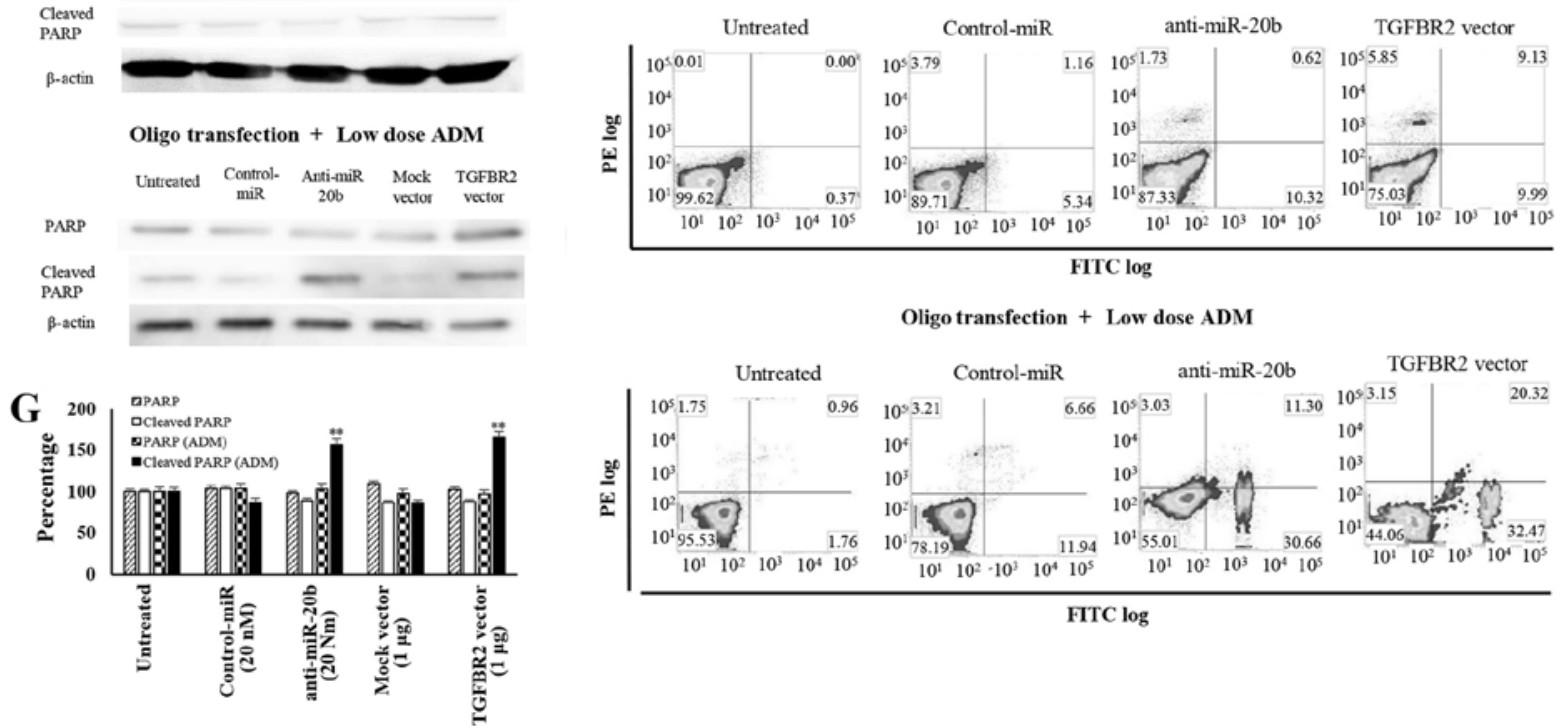

Figure 5. Anti-miR-20b and TGFBR2 expression vector treatment induces cell cycle retardation. Cells were untreated (A) or transfected with negative control $\mathrm{miR}(\mathrm{B})$, anti-miR-20b (C) and TGFBR2 expression vector (D), stained with propidium iodide and analyzed for cell cycle distribution. Histogram shows the mean values of each cell cycle phase $(\mathrm{E})$ : ${ }^{*} \mathrm{P}<0.05,{ }^{* *} \mathrm{P}<0.01$. Detection of cleaved PARP levels present in protein levels by immunoblot. Anti-miR-20b and TGFBR2 expression vector with low-dose adriamycin treatment induces apoptosis (F). The quantification of PARP and cleaved PARP protein (G). The above experimental results were analyzed for the presence of Annexin V (+)/PI (-) (early apoptosis) and Annexin V (+)/PI (+) (late apoptosis). SKES1 cells treated with anti-miR-20b and TGFBR2 expression vector and anti-miR-20b and TGFBR2 expression vector with low-dose adriamycin were stained with Annexin V-FITC/PI, and analyzed for cell apoptosis distribution (H).

or TGFBR2 vector-transfected cells $\left(385.5 \pm 16.9\right.$ cells $\left./ \mathrm{mm}^{3}\right)$ was significantly smaller than that following transfection with untreated $\left(1165.8 \pm 74.1\right.$ cells $\left./ \mathrm{mm}^{3}\right)$ and NC-miRNAtransfected cells $\left(1021.2 \pm 54.7\right.$ cells $\left./ \mathrm{mm}^{3}\right)$. These results suggest that the inhibition of miR-20b expression also leads to a reduction in the in vivo growth of ES cells. Immunohistochemical analysis revealed that the expression of TGFBR 2 and MYC in xenografted tumors was inhibited by transfection with the anti-miR-20b and TGFBR2 expression vectors (Fig. 6B). The number of cells positive for TGFBR2 expression was significantly increased in mice inoculated with the anti-miR-20b $\left(199 \pm 21.8\right.$ cells $\left./ \mathrm{mm}^{2}\right)$ or TGFBR2 expression vector-transfected cells $\left(230.7 \pm 45.7\right.$ cells $\left./ \mathrm{mm}^{2}\right)$ compared to untreated $\left(65.6 \pm 10.6 \mathrm{cell} / \mathrm{s} / \mathrm{mm}^{2}\right)$ or Control-miR transfected cells $\left(93.8 \pm 16.8\right.$ cells $\left./ \mathrm{mm}^{2}\right)(\mathrm{P}<0.01 ;$ Fig. $6 \mathrm{C})$. The number of MYC-expressing cells was significantly reduced in mice inoculated with anti-miR-20b $(113.2 \pm 26.2$ cells/ $\left.\mathrm{mm}^{2}\right)$ or TGFBR2 vector $\left(76.9 \pm 26\right.$ cells $\left./ \mathrm{mm}^{2}\right)$ transfected cells compared to untreated $\left(244.6 \pm 62.1 \mathrm{cells} / \mathrm{mm}^{2}\right)$ or Control-miR-transfected cells $\left(219.6 \pm 42.5\right.$ cells $\left./ \mathrm{mm}^{2}\right)$ $(\mathrm{P}<0.01$; Fig. 6C).

\section{Discussion}

miRNAs are a group of small, non-coding RNAs that regulate protein-coding genes (16). To determine the miRNA-mRNA relationship in ES, we carried out genome-wide miRNA array and cDNA array analyses.

Our array data regarding miRNA deregulation revealed increased aberrant miR-20b expression in ES cells compared to that in hMSCs. Several studies have shown that miR-20b is upregulated and involved in potential targets for cancer therapy, diagnosis and prognosis (5-7). However, the biological characteristics of miR-20b in ES cells remained unclear. Our data demonstrated that miR-20b expression was upregulated in ES cell lines, and we conducted genome-wide mRNA profiling by cDNA array to reveal the possible targets of miR-20b in ES cells.

The cDNA array data showed that TGFBR 2 mRNA expression was decreased in all five ES cell lines. Furthermore, alignment analysis indicated a possible match between miR-20b and the $3^{\prime}$-UTR of TGFBR2. TGFBR2 is among the most frequently transformed tumor-suppressor genes in human 

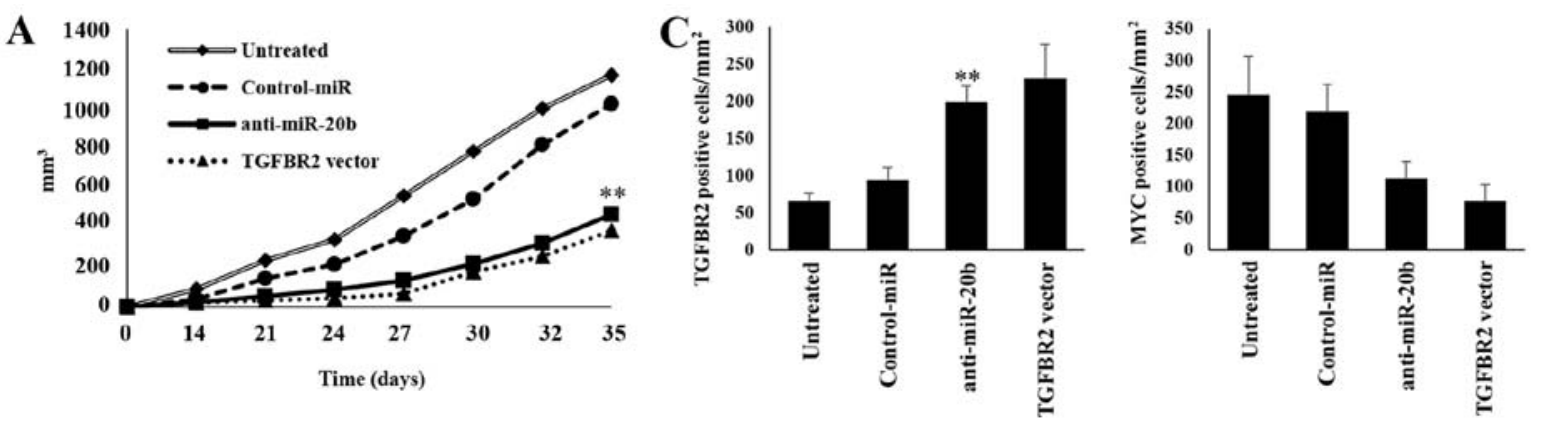

\section{B}
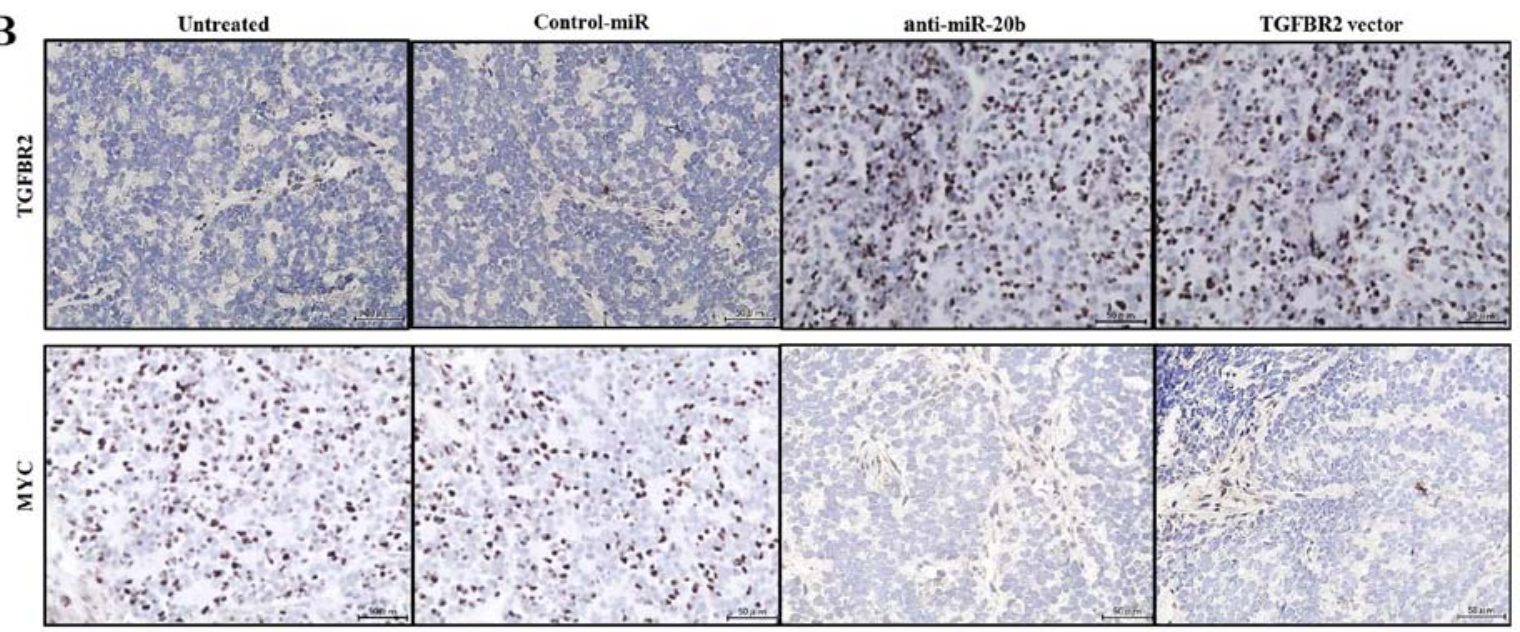

Figure 6. Suppression of ES tumor growth by anti-miR-20b and TGFBR2 vector in vivo. (A) Four groups of mice inoculated with: i) untreated SK-ES-1 cells $(\mathrm{n}=7)$; ii) transfected with negative control-miR $(\mathrm{n}=7)$; iii) transfected with anti-miR-20b $(\mathrm{n}=7)$; and iv) transfected with TGFBR2 expression vector ( $\mathrm{n}=7$ ). Tumor volumes were measured every 3 days during treatment. ${ }^{* *} \mathrm{P}<0.01$. (B) Immunohistochemistry image analysis of TGFBR2 and MYC following transfection of anti-miR-20b and TGFBR2 vector. (C) Number of TGFBR2- and MYC-positive cells per unit area.

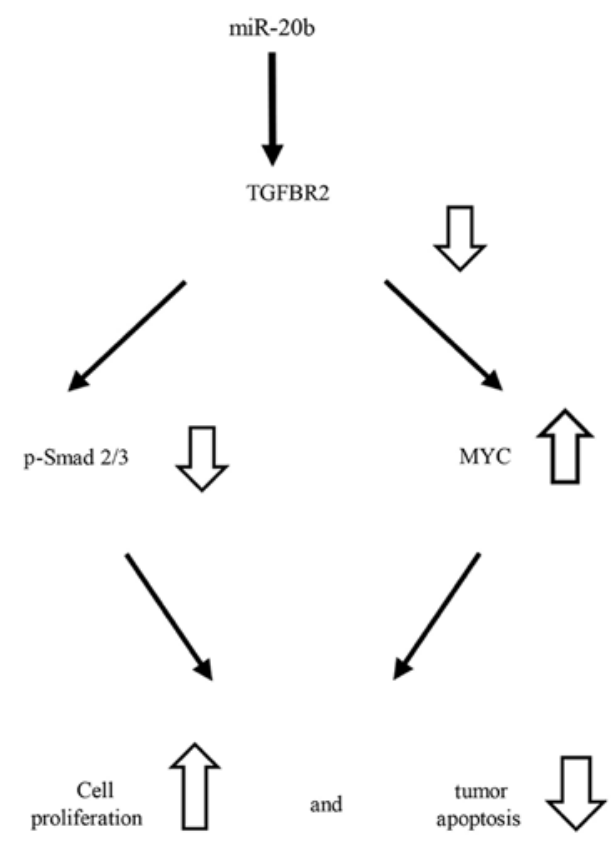

Figure 7. A flowchart miR-20b axis in the attenuation of TGFBR2 signaling in Ewing's sarcoma cell lines.

cancers, driving actuation of the MYC pathway and increasing cell survival (12). Several studies have suggested that TGFBR2 plays an important role in the complex network of oncogenes by regulating cellular transformation. Downregulation of TGFBR2 has been observed in colon cancer (17) and hepatocellular carcinoma (18). Our data in ES cells agree with the results of previous studies showing that decreased TGFBR2 may contribute to malignant potential.

Although miR-20b may affect gene expression, we regarded TGFBR2 as the target of miR-20b in ES cells. Several studies have detected several target genes of miR-20b, including phosphatase and tensin homolog and matrix metalloproteinase $2(19,20)$. Our cDNA array data indicated that TGFBR 2 was the only miR-20b-target gene and was uniformly decreased in all five ES cell lines. BLAST and TargetScan analyses further supported that TGFBR2 is the tentative target of miR-20b. miR-20b may decrease TGFBR 2 expression through an indirect pathway; however, TGFBR2 mRNA degradation was increased even after inhibiting de novo mRNA transcription. miR-20b did not inhibit TGFBR2 mRNA expression in mutant miR-20b-transfected cells. Therefore, miR-20b may regulate TGFBR2 mRNA stability.

We further examined the role of miR-20b in the regulation of its possible target gene TGFBR2 and changes in the malignancy potential of ES cell lines. Transfection with the anti-miR-20b and TGFBR2 expression vectors into ES cells enhanced $\mathrm{p}-\mathrm{Smad} 2 / 3$ and repressed MYC protein, indicating that the restoration of TGF- $\beta$ signaling controls MYC expression. It has been reported that MYC expression is significantly downregulated in response to TGF- $\beta$ signaling and that the Smad pathway is an upstream regulator of MYC expres- 
sion (21). Our data suggest that downregulatory mechanisms of MYC expression downstream of TGF- $\beta$ signaling and of TGFBR2 expression via miR-20b may occur in ES cells.

Our results regarding the cell cycle revealed that miR-20b promoted the proliferation of ES cells by inducing cell cycle retardation. These results are consistent with the MYC and p-Akt downregulation in anti-miR-20b and TGFBR2 expression vector-transfected cells. Previous reports demonstrated that Smad2/3 phosphorylation via TGFBR2 activation is necessary for controlling the G1/S checkpoint (21). p15, p21 and p27 expression increased as TGFBR2 was expressed. As a result, the progression of the cell cycle was delayed. TGFBR2 regulates the G1/S transition and cell growth, and restoration of TGFBR2 leads to retardation of the cell cycle in the G1 phase (22). Thus, upregulation of miR-20b may influence the cell cycle progression of ES cells through miR-20b-mediated regulation of TGFBR2.

Since TGFBR2 is a major negative factor in MYC expression in ES, the repression of TGFBR2 by miR-20b may play an important role in cell growth (Fig. 7). Dysfunction of Smad2/3 phosphorylation through TGFBR2 may upregulate MYC and lead to cell proliferation and an anti-apoptotic status. Thus, phosphorylation of Smad2/3 by TGFBR2 in ES cells may induce downregulation of MYC, resulting in the deceleration of ES cell growth.

Moreover, the suppression of miR-20b led to inhibition of ES tumor growth in vivo. In accordance with the in vitro data, the xenograft model of ES also suggested that the reduction in miR-20b expression could inhibit ES tumor growth in vivo via TGFBR2 restoration.

In conclusion, the present study revealed an inverse correlation in miR-20b and TGFBR2 in ES cells. We previously investigated the aberrant expression of MYC in ES cell lines (23). Based on our results, MYC may be repressed by phosphorylation of Smad via suppressing the expression of TGFBR2. As a result, appropriate expression of TGFBR2 can regulate $\mathrm{MYC}$ expression and control the cell cycle and tumor survival of ES. The present data regarding the link between miR-20b, TGFBR2 and MYC in ES cells may improve the understanding of the oncogenesis of ES and provide a novel strategy for clinical application.

\section{Acknowledgements}

The present study was supported in part by the fund of the National Cancer Center Research and Development (26-A-4) and the Grants-in-Aid for Scientific Research (nos. 15K10451, $16 \mathrm{~K} 10866$ and 16K20063) from the Japan Society for the Promotion of Science.

\section{References}

1. Esiashvili N, Goodman M and Marcus RB Jr: Changes in incidence and survival of Ewing sarcoma patients over the past 3 decades: Surveillance Epidemiology and End Results data. J Pediatr Hematol Oncol 30: 425-430, 2008.

2. Womer RB, West DC, Krailo MD, Dickman PS, Pawel BR, Grier HE, Marcus K, Sailer S, Healey JH, Dormans JP, et al: Randomized controlled trial of interval-compressed chemotherapy for the treatment of localized Ewing sarcoma: A report from the Children's Oncology Group. J Clin Oncol 30: 4148-4154, 2012.
3. Sayed D and Abdellatif M: MicroRNAs in development and disease. Physiol Rev 91: 827-887, 2011.

4. Lima RT, Busacca S, Almeida GM, Gaudino G, Fennell DA and Vasconcelos MH: MicroRNA regulation of core apoptosis pathways in cancer. Eur J Cancer 47: 163-174, 2011.

5. Li S, Qiang Q, Shan H, Shi M, Gan G, Ma F and Chen B: MiR-20a and $\mathrm{miR}-20 \mathrm{~b}$ negatively regulate autophagy by targeting RB1CC1/FIP200 in breast cancer cells. Life Sci 147: 143-152, 2016.

6. Danza K, Silvestris N, Simone G, Signorile M, Saragoni L, Brunetti O, Monti M, Mazzotta A, De Summa S, Mangia A, et al: Role of miR-27a, miR-181a and miR-20b in gastric cancer hypoxia-induced chemoresistance. Cancer Biol Ther 17: 400-406, 2016.

7. Yamaguchi T, Iijima T, Wakaume R, Takahashi K, Matsumoto $H$, Nakano D, Nakayama Y, Mori T, Horiguchi S and Miyaki M: Underexpression of miR-126 and miR-20b in hereditary and nonhereditary colorectal tumors. Oncology 87: 58-66, 2014.

8. Chowdhury S, Ammanamanchi S and Howell GM: Epigenetic targeting of transforming growth factor $\beta$ receptor II and implications for cancer therapy. Mol Cell Pharmacol 1: 57-70, 2009.

9. Ikushima $\mathrm{H}$ and Miyazono K: TGFbeta signalling: A complex web in cancer progression. Nat Rev Cancer 10: 415-424, 2010.

10. Yu Y, Kanwar SS, Patel BB, Oh PS, Nautiyal J, Sarkar FH and Majumdar AP: MicroRNA-21 induces stemness by downregulating transforming growth factor beta receptor 2 (TGF $\beta R 2$ ) in colon cancer cells. Carcinogenesis 33: 68-76, 2012

11. Mishra S, Deng JJ, Gowda PS, Rao MK, Lin CL, Chen CL, Huang $T$ and Sun LZ: Androgen receptor and microRNA-21 axis downregulates transforming growth factor beta receptor II (TGFBR2) expression in prostate cancer. Oncogene 33: 4097-4106, 2014.

12. Zhu X, Ozturk F, Liu C, Oakley GG and Nawshad A: Transforming growth factor- $\beta$ activates c-Myc to promote palatal growth. J Cell Biochem 113: 3069-3085, 2012.

13. Kawano M, Tanaka K, Itonaga I, Ikeda S, Iwasaki $\mathrm{T}$ and Tsumura H: microRNA-93 promotes cell proliferation via targeting of PTEN in Osteosarcoma cells. J Exp Clin Cancer Res 34: 76, 2015.

14. Tanaka K, Kawano M, Itonaga I, Iwasaki T, Miyazaki M, Ikeda S and Tsumura H: Tumor suppressive microRNA-138 inhibits metastatic potential via the targeting of focal adhesion kinase in Ewing's sarcoma cells. Int J Oncol 48: 1135-1144, 2016.

15. Kawano M, Tanaka K, Itonaga I, Iwasaki T and Tsumura H: MicroRNA-301a promotes cell proliferation via PTEN targeting in Ewing's sarcoma cells. Int J Oncol 48: 1531-1540, 2016

16. Lu J, Getz G, Miska EA, Alvarez-Saavedra E, Lamb J, Peck D, Sweet-Cordero A, Ebert BL, Mak RH, Ferrando AA, Downing JR, et al: MicroRNA expression profiles classify human cancers. Nature 435: 834-838, 2005.

17. Yu M, Trobridge P, Wang Y, Kanngurn S, Morris SM, Knoblaugh S and Grady WM: Inactivation of TGF- $\beta$ signaling and loss of PTEN cooperate to induce colon cancer in vivo. Oncogene 33: 1538-1547, 2014.

18. Mamiya T, Yamazaki K, Masugi Y, Mori T, Effendi K, Du W, Hibi T, Tanabe M, Ueda M, Takayama T, et al: Reduced transforming growth factor-beta receptor II expression in hepatocellular carcinoma correlates with intrahepatic metastasis. Lab Invest 90: 1339-1345, 2010.

19. Wang B, Yang J and Xiao B: MicroRNA-20b (miR-20b) promotes the proliferation, migration, invasion, and tumorigenicity in esophageal cancer cells via the regulation of phosphatase and tensin homologue expression. PLoS One 11: e0164105, 2016.

20. Park SL, Cho TM, Won SY, Song JH, Noh DH, Kim WJ and Moon SK: MicroRNA-20b inhibits the proliferation, migration and invasion of bladder cancer EJ cells via the targeting of cell cycle regulation and Sp-1-mediated MMP-2 expression. Oncol Rep 34: 1605-1612, 2015.

21. Zhao HW, Li YW, Feng R, Yu JB, Li J, Zhang Y, Li JC and Wang YX: TGF- $\beta /$ Smad2/3 signal pathway involves in U251 cell proliferation and apoptosis. Gene 562: 76-82, 2015.

22. Choi J, Park SJ, Jo EJ, Lee HY, Hong S, Kim SJ and Kim BC: Hydrogen peroxide inhibits transforming growth factor$\beta 1$-induced cell cycle arrest by promoting Smad3 linker phosphorylation through activation of Akt-ERK1/2-linked signaling pathway. Biochem Biophys Res Commun 435: 634-639, 2013.

23. Kawano M, Tanaka K, Itonaga I, Iwasaki T and Tsumura $\mathrm{H}$ : c-Myc represses tumor-suppressive microRNAs, let-7a, miR-16 and miR-29b, and induces cyclin D2-mediated cell proliferation in Ewing's sarcoma cell line. PLoS One 10: e0138560, 2015. 